lumbar region, which was puckered and contracted. Some small hard bodies (calculi) could also be felt deep seated beneath the integuments in this region, near the spine. His urine contained a considerable amount of pus, and was of specific gravity 1010. His general health was tolerably good; his skin generally was dark.

R Tincturæ ferri sesquichloridi 3 iss; infusi quassiæ 3 viij. M. Fiat mist. Capiat $z_{j}$ ter die.

Full diet was ordered.

This plan was continued for a fortnight, without much improvement.

September 17th. He was ordered-

R Acidi gallici gr. v; confectionis rosæ q. s. ut fiat pilula bis die sumenda.

He was ordered to have half a pint of porter daily.

Both his general health and the state of his urine improved considerably under this treatment. The latter, however, still contained a small quantity of pus.

October 23rd. He was made an out-patient, at his own request

Whilst in hospital, an incision was made in his back, and several small round calculi removed from beneath the lumbar fascia. Others, amounting in all to about thirty, worked themselves out through the wound, which subsequently healed.

He continued an out-patient up to January 1st, 1859, when be was readmitted into the house. His urine now contained little or no pus. But it would be tedious to relate the further progress of the case in detail. He was again put on the tincture of muriate of iron; subsequently, mineral acids and quinine, with generous diet. An issue was made in his loins, but he could not bear it. His urine at one time was perfectly free from pus, or any other sediment; but it subsequently returned. He left again in February; presented himself for the last time on April 30th, bringing thirty or forty more small calculi, which had been discharged from his back; and on May 28 th he died, with symptoms of uræmic poisoning, under the care of Mr. Morley, through whose kindness I enjoyed the opportunity of being present at the post mortem examination. A small slough was passed per urethram a few days before death.

Autopsr, forty-eight hours after death. There was considerable emaciation. All rigidity had passed off. The heart was soft and flabby, otherwise apparently healthy; it was not opened. The liver was paler than natural; the right lobe was enlarged. The right kidney was more than twice the natural size; on section, it was pale, soft, and fatty, having undergone the fatty degeneration; the tubular portion was very indistinct its capsule was adherent throughout. In the situation of the left kidney, which was not to be recognised, was a mass of almost cartilaginous hardness, from which, on pressure, a quantity of pus flowed. On section, it was seen to be riddled with abscesses, and contained numerous small calculi, like those which had been extracted from his back; and a few larger ones, which took the shape of the part in which they lay. Some of the smaller ones were firmly imbedded lower

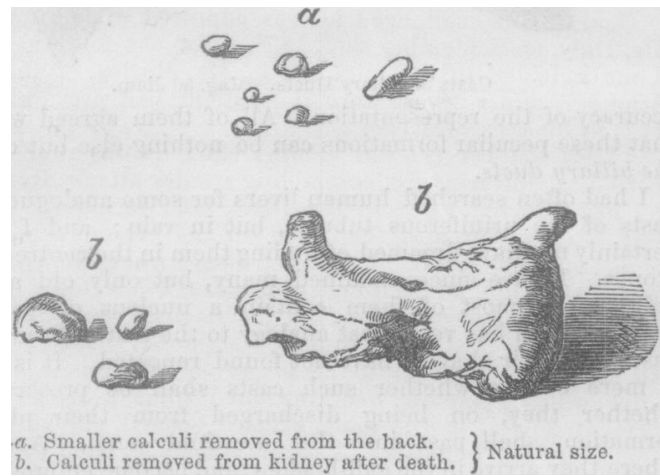

down in the course of the ureter, as if on their way towards the bladder. When this hard mass was removed, one of the ribs was found to be denuded of periosteum. The right suprarenal capsule was apparently healthy; the left was not to be seen; nor was the ureter on this side at all distinguishable. The bladder was empty and contracted, healthy. The other organs (head not examined) were healthy. The calculi were composed chiefly of uric acid, with a small proportion of earthy phosphate.

\section{OHitional Communitations.}

\section{ON THE PATHOLOGY AND TREATMENT OF GALL-STONES.}

By J. L. W. Thudichuir, M.D.

[Read brfore the Aledical Society of London, October 17th, 1859.]

THose who engage in post mortem examinations of the human body have not rarely an opportunity afforded to them of examining concretions in the gall-bladder or gall-ducts. These already for several centuries have engaged the attention of the learned, or roused the wonder of the curious. To either it must be a subject of astonishment that a large number of solid hard bodies should be met with, filling almost the entire cavity of a receptacle destined to hold a mild and innocuous fluid, and that yet there should have been no symptoms during the life of the individual who carried those concretions in his biliary organs, indicating their presence. There is no doubt that, in the majority of these cases, life came to a premature termination by some general disorder, not indeed produced by those foreign bodies, nor having any direct connection with the mere fact of their presence, but perhaps linked, however remotely, to the cause that originally produced them. Gall-stones, as we meet them in the dead body, are the residue of a pathological process, the nature of which it is then mostly too late to assertain by direct inquiry.

To many mortals, on the other hand, gall-stones prove by no means mild and innocuous customers, and by frequent and painful effects only too sensibly remind them of their existence. These painful and sometimes fatal attacks the physician is called upon to relieve, to heal, or, as the case may, be to prevent Like the morbid anatomist, the practical physician has to deal with the consequences of a disease which is itself not the object of his immediate care. We may compare both investigators to the geologist, whose inquiry into the history of an outburnt volcano has to be carried on upon the ashes and the lava, or other rocky products, and upon the large features of their conformation. It is impossible to observe their direct genesis, - the fire that produced them is long since extinct.

An inquiry into the pathology of gall-stones is, therefore, mostly an inquiry into causes so remote and obscure, that the difficulties encountered in such a proceding are amongst the greatest which medical science has to battle with. Accordingly, our positive knowledge of those causes at present amounts to very little or nothing; surmise has taken the lead, and has assigned various causes, such as the predisposition of age and $\mathrm{sex}$, the individual disposition or diathesis, which, like all diatheses, is but a circumlocution of the mystery. Among the alleged causes, we find climate, excess of rest and long sleeping, and unwholesome food, and a variety of other indifferent circumstances, which, on a stringent scrutiny, must stand aside as incompetent.

In thus acknowledging at the outset, that the primary disease which causes gall-stones is quite unknown, that it is not even ascertained whether the disorder is a local disease of the livercells, or a decomposition of the bile, analogous to those decom. positions of the urine which produce the uric acid calculus, or a general disorder of the blood, or of the portal blood in particular, I do not mean to surrender all hope for a fair solution of the question in a comparatively short time. The study of the structure and composition of gall-stones has already taught us much; and, so soon as the chemistry of the liver and bile shall be well understood, we shall be in a position to approach the problem from both sides, during life and after death, by physiological research and experiment, as well as by the anatomical and chemical examination of the dead body.

Having instituted some inquiries in that direction, I have made such experiences as my limited opportunities and materials did admit of. One observation at least has been to me a fruitful source of reflection and information, and in communicating it to you linked to a few others of more practical import, I have consulted not only my wish to contribute an instalment towards the great capital of science which we are accumulating, but also my desire of profiting by the opinions and the advice of my professional brethren.

CASE I. This case is one of several in which Mr. Holmes, of St. George's Hospital, kindly afforded me an opportunity of examining the livers and bile of deceased persons. The notes 
relative to the case were given to me from the register of St. George's Hospital, by Dr. G. G. Rogers.

"Sarah Paine, married, aged 60 , was admitted into St. George's Hospital, on June 23rd, 1858, under the care of Dr. Pitman. She had anasarca of the lower extremities, dysnnœa, and livid countenance. She stated that she had been ill six weeks, with pain at the chest and palpitation. Once or twice she spat a little blood. There was no history of rheumatism, but the action of the heart was violent, and a murmur could be heard at the apex, with prolongation of the first sound at the base. Digitalis was given with nitric ether and acetate of potass every six hours, and she was purged with calomel and jalap. This treatment relieved the dropsy, but the breathing grew more oppressed, amounting to orthopnoa. She got but little sleep, and was more harassed by cough. None of the urine could be saved for examination, but the cardiac symptoms were quite sufficient to account for the dropsy, and she had never been troubled with any diminution in the quantity of urine, pain in the loins, etc. As the breathing grew worse, a blister was applied between the shoulders; infusion of roses with dilute mineral acids being given to check the hæmoptysis. She sank within a week from the time of admission. (Signed) G. G. R."

"The examination of the body was instituted twenty-six hours after death. Externally, the body appeared in good condition. The subarachnoid space contained a good deal of fluid, and the brain was watery. Otherwise the cranial contents were natural. There was much fluid in both pleural cavities, and extensive adhesions in the right. The upper parts of both lungs were healthy, but both contained extensive extravasations of blood at their bases. This extravasation had consolidated all the base of each lung for the space of about an inch. The heart was of large size. The edges of the mitral valve were much thickened, especially on the auricular aspect, and the orifice so contracted, as only to admit the point of the forefinger. The aortic valves were also thickened, and very rigid. The ribs were much pressed in, apparently by tight lacing. The liver was (apparently) healthy, except that at its sharp edge a rounded mass, about the size of a walnut was found, which was of a semisolid consistence, and whitish colour, and contained the bags of numerous hydatids, attached to which, as I was informed (by Dr. 'Thudichum), the hooklets of the echinococcus were found in large numbers. 'The gall-bladder was large, and contained numerous gall-stones, but the duct did not seem obstructed. The spleen and suprarenal glands were healthy. The kidneys were healthy; except that there was a deep depression on the surface of the right, probably the mark of an old cyst. The abdominal aorta was extensively atheromatous. The ovaries were shrunken. The os uteri was closed by a plug of gelatinous lymph, and the eavity of the uterus contained some turbid fluid. (St. George's Hospital Register, 1858. No. 1623. P. M. E. No. 177.)

$$
\text { (Signed) }
$$

"T. Holares."

On examining the bile in the gall-bladder, I found it to consist of a homogeneous fluid, containing little colouring matter in solution, but a large amount of brownish-yellow colouring matter, together with many crystals of cholesterine were suspended in it. The bile and colouring matters dissolved in alcohol, yielding a yellow solution, which was separated from mucus and epithelium by filtration. The alcohol was distilled off, and the residue tested with sugar and sulphuric acid. But no colour-test could be obtained with the greatest care, and although the test was repeated several times: there was not a trace of biliary acid in this fluid. But it contained some margaric and stearic acids, which, together with the cholesterine, crystallized from the concentrated extract.

The gall-stones were sixteen in number. On dividing the largest one into two halves, I found a large nucleus of brown pulpy matter, which could easily be removed with the point of a knife, or washed away with a brisk stream of water from a wash-bottle or so-called blow-bottle. When collected in a white china dish, the matter appeared to be composed of thread-like fibres, of different diameters, some a quarter of an inch long; some shorter pieces were one-sixteenth of an inch in thickness. All were cylindrical, as if moulded in tubes; many had branches, others were divided dichotomically. The thinnest portions had a diffuse broom-like end, as if the matter had not had time or quality to solidify in the tubular form, or as if it had solidified in a bag-like enlargement of the cylinder, in which the rest of the cast was moulded. The matter composing these productions was granular, without a trace of crystallization of any kind; was purely yellow in the thinnest branches, but became the darker brown, the thicker the forms grew. All further de936 scription becomes at once unnecessary, by the faithful representations of the more striking portions which I have caused to be executed by Mr. Lens Aldous. Some forty or fifty medical gentlemen, to whom I had an opportunity of submitting both the specimens and their drawings, have borne witness to the

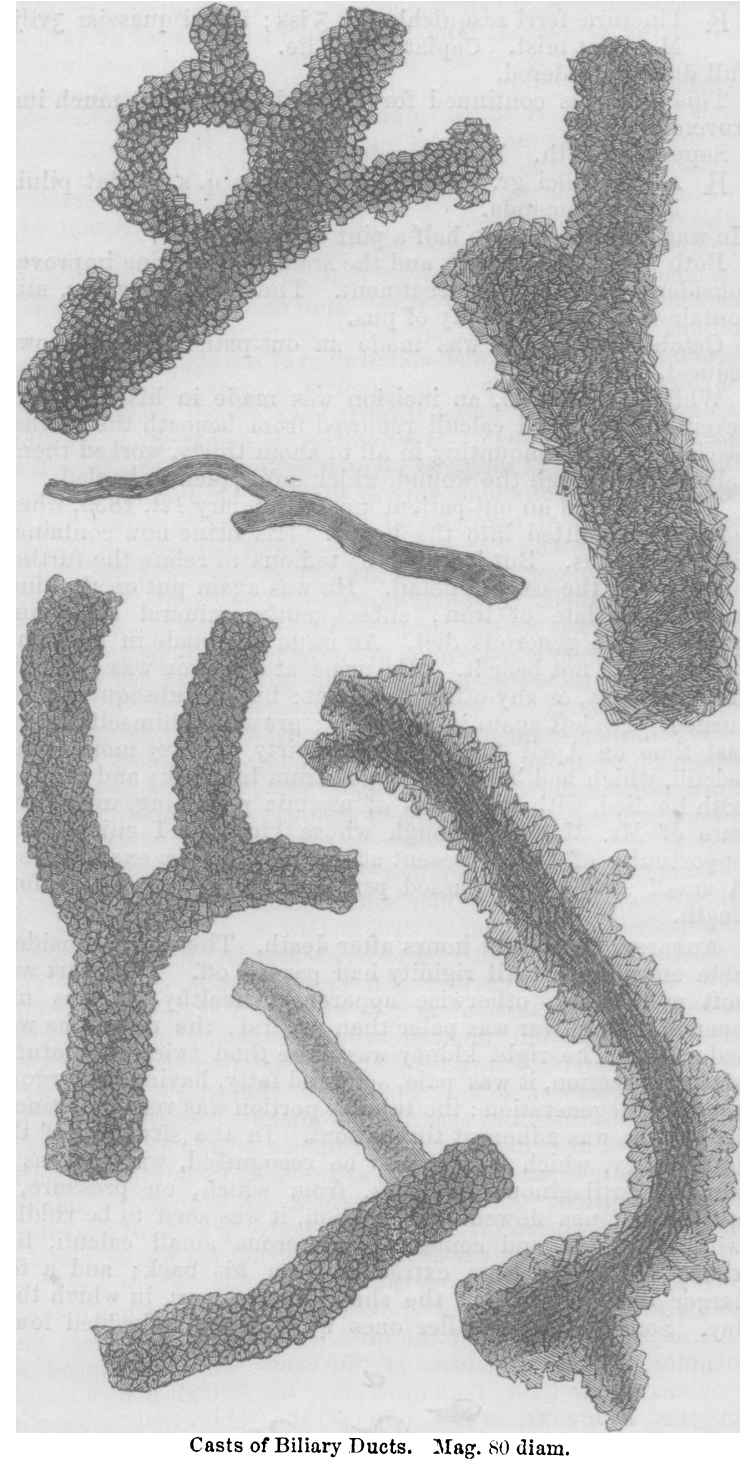

accuracy of the representation. All of them agreed with me that these peculiar formations can be nothing else but casts of the biliary ducts.

I had often searched human livers for some analogue to the casts of the uriniferous tubules, but in vain; and I should certainly not have dreamed of finding them in the centre of gallstones. I have since examined many, but only old and dry gall-stones; most of them contain a nucleus of colouring matter, which has very great analogy to the matter forming the casts, but their shape I have not found repeated. It is indeed a mere chance whether such casts shall be preserved whether they, on being discharged from their place of formation, shall pass into the intestinal canal. And even where they arrive in the gall-bladder, and become entangled and covered with cholesterine, evidently a secondary occurrence, it is again a mere chance whether they shall not be so sur. rounded and interlaced by cholesterine crystals, that, on breaking the stone, they must necessarily come to pieces. They are so fragile, that the mere weight of a thin glass cover, as used for microscopical preparations, was sufficient to crush the thinner ones. When shaken in the watch-glasses, in which I kept them, moistened with glycerine, the mere friction of one against the other would damage and disintegrate the most characteristic feature. About half the number of gall-stones, and 
among them some large sized ones, although containing a good brown nucleus, yet did not admit of the separation of characteristic casts, although among the débris I could distinguish fragments of casts with ease and certainty.

The material of the casts was not chemically homogeneous. Alcohol extracted a yellow portion; another portion, probably bilifulvine, remained. Some bad a peculiarly ragged or variably projecting outline, which made me examine for cylindrical epithelial cells; but, however great the probability that such cells might adhere to the circumference, or enter into the body of the casts, being epithelium proper to the biliary ducts, certain it is that no such formations could be identified.

Numerous are the reflections suggested by this observation. The pathological process which effected the effusion of a congealing colouring matter into the biliary ducts was probably an acute one; if it involved only a portion of the liver, it probably presented itself at the time under the form of a true bilious attack, that is to say, a derangement of digestion, fever, headache, and a yellowish colouration of the skin, and albuginea of the eyes. If the process involved the greater part or the whole of the biliary ducts, it must have shown itself under the form of acute jaundice. It is not impossible, or rather probable, that those forms of idiopathic jaundice which hitherto have been ascribed to spasmodic closure of the duct, or to failing secretory activity, a sort of paralysis of the liver may hereafter find an explanation by the discovery of a real and material obstruction of the passages of the bile by formations similar to those which I have described. Frerichs saw a case of jaundice coming to a favourable crisis by the discharge of brown flakes and threads, which were very conspicuous upon the light coloured fæces, and ushered in the reappearance of the ordinary brown colour of the evacuations.

The branched calculi of Plater, and the branched gall-stones which Fauconneau-Dufresne in one instance discovered in the finer ramifications of the biliary ducts, have perhaps some analogy to the casts of the bile-ducts brought under your notice.

In continuing for a moment the consideration of the etiology of gall-stones, I beg to offer some fragmentary speculations on the possible or probable circumstances which may produce gall-stones. It remains yet to be ascertained whether a foreign body in the gall-bladder can simply predispose the cholesterine to crystallise upon it, or whether a change in the composition of the bile must precede that separation, as alkaline urine must precede the phosphatic incrustation of foreign bodies in the urinary bladder. There are cholesteric calculi containing pins, flukes, or ascarides as nuclei. I shall have an opportunity of bringing some experiments on this question before you on a subsequent occasion, as it seems to admit of a satis factory solution. At present we must allow, that the nucleus being given, whether it be a foreign body, or casts of the biliary ducts, or amorphous precipitated colouring matter, or blood, or cast off epithelium, or even the problematical mucus-the subsequent crystallization of cholesterine may be either a conse quence of the effects of the nucleus upon the bile and gallbladder, or a continuation of the cause which produced the nucleus. The casts may have acted as foreign bodies simply, or the liver.cells, which effused the abnormal congealing matter, may have thrown out an excess of cholesterine, or bile incapable of holding the ordinary quantity of cholesterine in solution.

As there are gall-stones which possess no tangible nucleus, but consist throughout of pure pearl-white cholesterine, the circumstances under which cholesterine may crystallize from bile, are particularly deserving of investigation. Excess of cholesterine merely, which Chevreul thought to have found, though a very likely thing to occur, is in itself not sufficient to explain its precipitation; a change or destruction of its solvent taurocholic acid, on its passage through the ducts and bladder, seems essential. This change may be produced by specific disease of the liver-tissue, by retention of bile and subsequent endosmotic changes, or by decomposition of bile from any cause. Bile, whether stagnating in the human body, or in a glass bottle, on decomposition setting in, gives up its choles. terine in a crystallised form.

This decomposition of the solvent of cholesterine, once set up in the gall-bladder, continues, like ammoniacal urine in the urinary bladder, to contaminate all fresh portions of bile poured out into the gall-bladder, and to precipitate their cholesterine, and often their colouring matter upon the centres of attraction. In this manner are formed the layers of gallstones, which in their concentricity exhibit the periodical growth of the concretions. Mixed with the cholesterine layers are patches or layers of colouring matter, namely, bilifulvine and biliverdine, in various proportions and shades. Bistearate of lime in star-like crystallized small masses, and a little phosphate of lime mixed with shrivelled cylindrical epithelial cells, make up the list of constituents which we observe in the greatest number of ordinary gall-stones. Concretions, consisting of pigment, or inspissated bile, or of phosphate and carbonate of lime, have a different pathological, and by their rarity, less practical significance.

As a proof that the above considerations are at least in part based upon personal observations, I will mention the following cases as having afforded the starting point for my reflections.

CASE II. In a female, dead from pulmonary phthisis, I found a globular gall-stone in the bladder, suspended in green thin bile. The concretion was white, like mother-o'-pearl, and there was no trace of a coloured nucleus. The whole seemed to consist of cholesterine, mixed with a trace of bistearate of lime, both uncoloured. It had not caused any symptoms during life.

CASE IIr. In the liver of a boy, dead from general tuberculosis, with tubercles in all the organs (including the muscular substance of the heart, a very rare occurrence), I found lumps of mere inspissated bile and epithelium, formed in cysts, which, although communicating with afferent biliary ducts, yet had no outlet, and were evidently enlargements of a portion of the obliterated gall-duct.

The symptoms produced by gall stones after their formation are so very characteristic and well known, that a repetition of general facts is uncalled for. Most of the lasting and obstinate symptoms centre in pain, due to inflammation or ulceration of the gall-bladder, or adjacent portions of liver or intestine. Distinct from these rarer chronic effects, are the more common manifestations of the passage of gall-stones into or through one or other of the ducts. The typical nature of these attacks mostly ensures their diagnosis. The pain and sickness, the band round the lower part of the chest, and other distinctly referred local sensations, indicate a gall-stone in the cystic duct. It may lodge there and close it for ever, and such an occurrence is not the worst issue of the disease. But when the gall-stone passes into the common duct, jaundice and vomiting, and more rarely a partial or entire absence of biliary colouring matter from the freces, lead our diagnosis. In this manne small concretions may be got rid of; larger concretions, up to an inch in length or diameter, are more rarely passed in this manner. So far as I know, there is, however, no case on record of a person, who during life suffered well authenticated attacks of passages of gall-stones, and was after death found not to harbour some concretion in his biliary passages.

Gall-stones not rarely cause inflammation of the gallbladder und adjacent portion of the liver. I have seen a circumscribed inflammation proceed to the peritoneal convex surface of the liver, and cause adhesion to the anterior $a b$ dominal parietes. When the exudation is more copious, and the process less chronic, abscess is sometimes formed, which happily mostly empties itself into the cavity of the duodenum more rarely into some other portion of the intestinal cavity, or into the peritoneal cavity, and in this case with rapidly fatal effects. In some extremely rare cases the abscess proceeds alo urachus into the urinary bladder. A case in which the abscess opened into the duodenum has fallen under my observation. The patient discharged suddenly with the greatest relief a quantity of purulent fluid, mixed with the pills, which his attendants believed to have passed unchanged through his body. They were evidently gall-stones, but had been poured away before I could examine them. Even without this ponfirmation, the case left no room for doubt.

The following case, which I had an opportunity of treating for several years, and watching to its conclusion, will be an ap propriate introduction to the important question of treatment. It shows that gall-stones may exist in the gall-bladder during forty years, produce recurrent attacks, and yet, with prudent living and medical assistance, the patient may live to eighty.

CASE IV. In the year 1853, I was requested to attend a gentleman, aged 75 , who, after having enjoyed excellent health for many years, was suddenly taken ill with the usual symptoms of the passage of gall-stones. On being questioned, he remembered having passed a gall-stone thirty-five years ago, which had been found bodily in the evacuations. Some slighter symptoms made a continuance of other concretions in the gallbladder, during the whole time which had elapsed since that occurrence, very probable. With the use of opium and occa- 
sional large draughts of warm water when the sickness seemed greatest, he recovered from that attack. The gall-stones, no doubt, passed successfully, which, from the presence of severe jaundice, is more probable than that they receded and returned into the bladder.

In the following year, he had another and more severe attack. It is probable that in this attack the false passage was formed from the gall-bladder to the duodenum, which I discovered at the post mortem examination, and the cystic termination of which enclosed an impacted calculus. The attack terminated with the discharge of gall-stones, brown biliary matter, and subsequent bilious diarrhuea.

From that time he began to take the celebrated mixture of turpentine and ether, and with so much apparent benefit, that during the subsequent four years the old gentleman allowed few days to pass without taking from half a drachm to a drachm of the mixture.

At the beginning of last winter his general health began to fail. A settled pain in the region of the gall bladder indicated chronic inflammation there. Notwithstanding all suitable treatment, he sank and died, very nearly eighty years old.

The post mortem examination revealed a gall-bladder full of calculi as the primary disease. Exudative plastic inflammation pervaded all neighbouring tissues.

The gall-stones were pulpy outside, and seemed to be made up of soft brown colouring matters; in the centre they were made up of crystallised cholesterine. I shew the dried bladder, containing one hard stone enclosed in the false passage. The others are removed. By this softness the theoretical value of the ether and turpentine mixture assumes a sort of empirical confirmation; although, on strict scrutiny, the direct solvent action of these substances cannot well be understood.

In the simptomatic treatment of the passage of gall-stones we have to rely mainly unon opium, which is sometimes better borne in the form of pills than that of tincture. We must carefully guard the patient from an overdose of this drug, which he is even more apt to take on his own account, from the torturing pain he is suffering, than the practitioner is likely to prescribe it. Nevertheless, severe narcotism sometimes follows large doses of opium - when the pain which caused them to be riven suddenly ceases, from local causes. Such a case happened in Ireland some years ago, and was the subject of a painful legal inquiry. Happily, the life of the patient, who was a member of Parliament and a large estate proprietor, ended fatally only a few days after the severe narcotic symptoms had been successfully combated, so that the death and the narcotism stood in no immediate connection with each other. Whatever be our opinion, this case suggests caution.

Persons atllicted with dormant gall-stones should regulate their living in the most careful way possible, avoid all and every description of spirituous liquors, and let fruit and cereals, and light vegetables, enter largely upon their dietary.

In lecided cases of gall-stones in the bladder, where continuous symptoms or repeated passages of concrements are observed, the surgeons should consider the propriety of planning and performing an operation for the extraction of these foreion bodies, either in a direct manner, or by forming a biliary fistula, and adopting a lithotriptic proceeding.

The operation is much slighter than lithotomy. Compared to such operations upon the abdominal organs as extirpation of ovarian tumours, it sinks into insigrificance. If, for example, an incision was made a little above the lower edge of the liver, just largo enough to admit the forefinger into the peritoneal cavity, the gall-bladder might be explored, and the presence or absence of calculi determined with the greatest certainty and the least danger. If calculi are present, the gall-bladder is seized with a small hook, pulled into the abdominal wound, and fixed there withont being opened. In six days a good and passabie fistula will be established, which, after a fortnight or three weeks, will be sufficiently consolidated to admit of the introduction of an instrument, by which such soft calculi may easily be crushed. The calculi once removed, I malie no manner of doubt that the fistula would heal up by itself. I am certain that, with proper care and dexterity, surgeons might make a number of these operations successful, and have few failures; considering always that cases suitable for the opera. tion would perhap's be half as common as cases for lithotomy.

Before linowing the cause and origin of gall-stones a little better, prevention is out of the question. But general hygiene will here do as much as in other diseuses.

The above remarks may, perhaps, appear insignificant to gentlemen of longer experience, and the results of my researches small to those possessing more extensive opportunities than 938 myself. I hope that such of my hearers as enjoy either will make up my deficiencies. If, in full consciousness of these latter, I have nevertheless come forward, I have done so upon the principle that, however small our light, we ought not to put it under the bushel, but let it shine before the people. Should it be very dull, then I hope that my friends, in the discussion, will give it the benefit of a suitable trimming.

\section{PATHOLOGICAL AND THERAPEUTICAL CON. SIDERATIONS RELATIVE TO INFLAM- MATION AND FEVER.}

By C. Handfiemd Jones, M.B., F.R.S., Physician to St. Mary's Hospital.

In the following pages I shall attempt to give a rationale of the principal phenomena observed in inflammation and fever, with particular reference to recent neuro-pathological researches.

1. It is well known that the arteries of the abdominal and pelvic viscera, of the thoracic, and of the encephalon, are surrounded with nervous plexuses derived from the sympathetic system. The filaments of these nerves accompany the vessels to their finer ramifications, if not to their ultimate; and are distributed to their coats in no inconsiderable measure. In the limbs the arteries are accompanied by no such distinct sympathetic plexuses; the cerebro-spinal cords appear to take their place. By microscopic examination $I$ have found that nerve filaments, in all respects similar to those of the visceral sympathetic, are given off from the finer ramifications of the cerebro-spinal nerves. They are in all probability distributed much in the same manner as the visceral nerves above mentioned. As branches from the ganglia of the sympathetic join the spinal nerves, it is no more than might have been expected that they would appear again at the peripheral expansions of the latter. The external carotid artery is surrounded by a plexus which accompanies each of its divisions. These are, therefore, similarly circumstanced to the visceral arteries of the three great cavities, which might have been anticipated considering their distribution.

In structure, the sympathetic nerves are found to consist of two kinds :- white tubular fibres, evidently identical with cerebro-spinal; and nucleated nerve bands, peculiar to the ganglionic nerves. The latter are known as fibres of Remak, and their nervous character is denied by several authorities. Bidder and Volkmann describe another variety of fibres which may be distinguished as the "fine tubular," and which they regard as peculiar to the sympathetic system. These have not the double contour, and are but half the size of the ordinary cerebro-spinal. I agree with Todd and Bowman, in adopting Remak's view. However this be, it seems unquestionable that there are fibres in the sympathetic derived from the cerebrospinal system, as well as others which arise in the various ganglia belonging to it. Anatomically, therefore, the sympathetic is a compound nerve, partly independent, and partly an offset of the nervous system of animal life.

2. Physiological observation accords with the above data, and extends them. The connection of the ganglionic nerves with the tissues of the part to which they proceed is proved by the influence of stimuli acting upon these nerves, or their centres, to excite muscular contractions or glandular secretion; and by the occurrence of pain, or reflex movements, when irritation is set up in the mucous surfaces, or other parts. The movements of the intestines and heart are unquestionably capable of being influenced by stimuli applied to their sympathetic nerves, or the related ganglia. The flow of gastric juice, of bile, and of pancreatic fluid, is produced by the stimulation of the mucous surface of the stomach and duodenum by the food. This stimulation is reflected through their nerves upon the several glandular organs. An irritant purgative causes griping pain and increased peristaltic motion. Muscular contractions can be influenced, in parts supplied with ganglionic nerves, by impulses originating in the brain or spinal cord : and conversely, the states of organs supplied with ganglionic nerves make themselves felt in the cerebral and spinal nervous centres. The effect of emotions upon the heart and the intestines is notorious, as well as the influence of intestinal disorder in producing headache, amaurosis, or tinnitus aurium, and of worms in causing convulsions.

The control exercised by the sympathetic nerves upon the vessels they accompany is very apparent in blushing, and in many pathological phenomena which will be hereafter alluded 